\title{
Ultrafast Magnon Generation in an Fe Film on Cu(100)
}

\author{
A. B. Schmidt, ${ }^{1,2, *}$ M. Pickel, ${ }^{1,2}$ M. Donath, ${ }^{2}$ P. Buczek, ${ }^{3}$ A. Ernst,${ }^{3}$ V. P. Zhukov, ${ }^{4}$ \\ P. M. Echenique, ${ }^{5}$ L. M. Sandratskii, ${ }^{3}$ E. V. Chulkov, ${ }^{5}$ and M. Weinelt ${ }^{1}$ \\ ${ }^{1}$ Max-Born-Institut, Max-Born-Straße 2A, 12489 Berlin, Germany and Freie Universität Berlin, \\ Fachbereich Physik, Arnimallee 14, 14195 Berlin, Germany \\ ${ }^{2}$ Physikalisches Institut, Westfälische Wilhelms-Universität Münster, Wilhelm-Klemm-Straße 10, 48149 Münster, Germany \\ ${ }^{3}$ Max-Planck-Institut für Mikrostrukturphysik, Weinberg 2, 06120 Halle, Germany \\ ${ }^{4}$ Institute of the Solid State Chemistry, Russian Academy of Sciences, Pervomayskaya 91, 620219, Yekaterinburg, Russia \\ ${ }^{5}$ Donostia International Physics Center (DIPC), Departamento de Física de Materiales, UPV/EHU, and \\ Centro de Física de Materiales CFM - MPC, Centro Mixto CSIC-UPV/EHU, San Sebastián, 20018 San Sebastian, Spain
}

(Received 4 December 2009; revised manuscript received 19 August 2010; published 5 November 2010)

We report on a combined experimental and theoretical study of the spin-dependent relaxation processes in the electron system of an iron film on $\mathrm{Cu}(100)$. Spin-, time-, energy- and angle-resolved two-photon photoemission shows a strong characteristic dependence of the lifetime of photoexcited electrons on their spin and energy. Ab initio calculations as well as a many-body treatment corroborate that the observed properties are determined by relaxation processes involving magnon emission. Thereby we demonstrate that magnon emission by hot electrons occurs on the femtosecond time scale and thus provides a significant source of ultrafast spin-flip processes. Furthermore, engineering of the magnon spectrum paves the way for tuning the dynamic properties of magnetic materials.

PACS numbers: 78.47.J-, 73.20.At, 75.30.Ds, 79.60.-i

Magnons are the fundamental collective excitations of the electron system of magnetic materials. In collinear ferromagnets each magnon lowers the magnetization by $2 \mu_{B}$. Understanding the generation of such spin waves away from thermal equilibrium is necessary to develop a microscopic picture of electron relaxation processes $[1,2]$. The signature of magnon generation by hot electrons has been found in spin-polarized electron energy loss spectroscopy [3], high-resolution photoemission spectra [4], and inelastic tunneling spectroscopy [5]. However, these experiments do not address the fundamental problem of the time required to generate a magnon. This is, in particular, important since magnon emission by excited electrons is commonly viewed as a slow process, occurring within picoseconds [6]. In contrast, laser-induced magnetic phase transitions are reported to take place in the femtosecond range [7-9]. Though parameterized models now describe major properties of laser-induced demagnetization dynamics, a microscopic understanding of these processes is still under debate [10-12].

In this Letter we present a combined experimental and theoretical approach to study ultrafast electronic dynamics in magnetic systems. The system we consider is a 3 monolayer iron film on $\mathrm{Cu}(001)$, since theory predicts spin-wave effects to be strong in $\mathrm{Fe}[13,14]$. A precise two-photon photoemission (2PPE) experiment with spin, time, energy, and angular resolution allows us to detect characteristic properties in the spin, energy, and momentum dependence of the lifetime of excited states. Based on $a b$ initio calculations of the ground state and the magnetic excitation of the system as well as the evaluation of the electron lifetimes within a many-body treatment of the electron self-energy, we show that the observed properties are determined by electron-magnon interaction. Our most fundamental result is that the emission of magnons by hot electrons takes place on a femtosecond time scale. This implies that magnon emission must be considered an important contribution to femtomagnetic properties of $3 d$ ferromagnets.

In our 2PPE experiment we probe the spin-dependent decay mechanisms of photoexcited electrons occupying image-potential states [15]. This class of surface states is localized several angstroms in front of the surface and they are established as the model system of choice for the study of electron dynamics [16]. As sketched in Fig. 1(a), a first femtosecond laser pulse $\hbar \omega_{a}$ excites an electron from bulk states below the Fermi level $E_{F}$ into the unoccupied imagepotential-state band and from there a second pulse $\hbar \omega_{b}$ raises the excited electron above the vacuum level $E_{\mathrm{vac}}$. The photoelectrons are then selected by energy and their spin polarization is determined (for further details see Ref. [17]). Measuring the energy as a function of the momentum parallel to the surface $E\left(k_{\|}\right)$yields the first and second band $(n=1,2)$ with a two-dimensional parabolic dispersion [18] as depicted in Fig. 1(a). Their exchange splitting is a signature of the ferromagnetic order in the iron film at the measurement temperature of $90 \mathrm{~K}$ $\left(T / T_{C}=0.24\right)[19,20]$.

The lifetime $\tau$ of electrons photoexcited to the $n=1$ band is accessed by shifting the time delay between pump and probe pulse. Figure 1(b) features six of such traces recorded at fixed kinetic energy, parallel momentum, and 

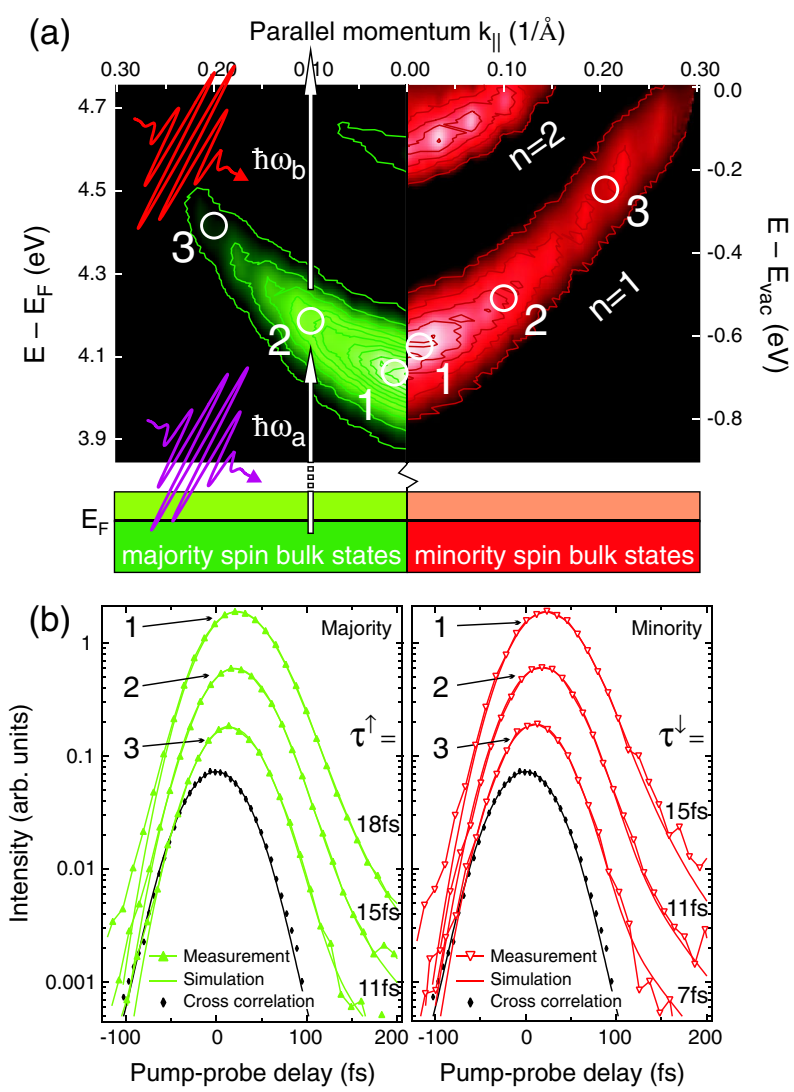

FIG. 1 (color online). (a) Dispersing first $(n=1)$ and second $(n=2)$ image-potential-state band as a function of momentum parallel to the surface. The intensity of the 2PPE signal in the majority- (left-hand side) and the minority-spin channel (righthand side) is presented in a contour plot with white indicating the maximum. The arrows depict the 2PPE process schematically. (b) Time-resolved 2PPE measurements of the $n=1$ imagepotential state taken at $k_{\|}$-values 1,2 , and 3 indicated in the top panel.

spin projection corresponding to points 1,2 and 3 in Fig. 1(a). The cross correlation of the Shockley surface state measured on clean $\mathrm{Cu}(111)$ (black diamonds) independently provides pulse durations and zero time delay. Simulations with optical Bloch equations (solid lines) yield the lifetimes $\tau^{\uparrow}$ and $\tau^{\downarrow}$ indicated close to the traces in Fig. 1(b) $[21,22]$. Where the shift of the time-resolved traces with respect to the cross correlation is smallest and the slope at positive delay is steepest, the lifetimes are shortest. Hence the time-resolved measurements demonstrate that minority-spin electrons decay faster than majority-spin electrons and that both, $\tau^{\uparrow}$ and $\tau^{\downarrow}$, decrease significantly with increasing momentum $k_{\|}$, and thus energy.

It is well established, that the decay of the excited image-potential electrons is dominated by inelastic electron-electron scattering, while electron-phonon scattering is negligible [16,23]. The three possible electronic decay channels are sketched in Fig. 2(a). Process 1 describes decay into empty bulk states. In this inelastic scattering process momentum and energy are conserved by an (a)

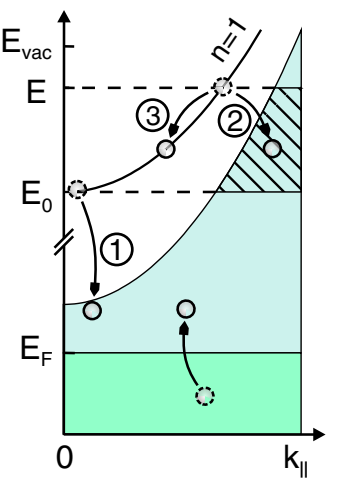

(b)

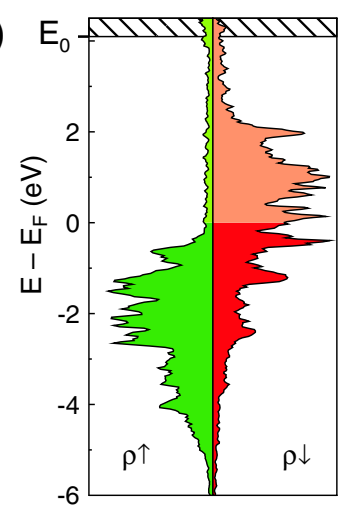

FIG. 2 (color online). (a) Schematic of the $n=1$ imagepotential state in the gap of the surface-projected bulk bands (filled areas). Inelastic decay processes of the image-potentialstate electrons via electron-hole pair creation are indicated (processes 1, 2, 3). (b) Calculated spin-resolved density of states for the surface layer of 3 monolayer iron on $\mathrm{Cu}(100)$.

electron-hole pair excited in the iron film [upward-pointing arrow in Fig. 2(a)]. A first-principles calculation of the density of states for the surface layer is shown in Fig. 2(b). The $d$ bands in the majority-spin channel are almost fully occupied, while the $d$ states in the minority-spin channel form a partially occupied band, exchange split from the majority bands by about $2.5 \mathrm{eV}$. It is this spin-dependent density of $d$ states, which determines the difference in majority and minority decay rates $\Gamma^{\dagger}=1 / \tau^{\uparrow}$ and $\Gamma^{\downarrow}=1 / \tau^{\downarrow}$ at the band minimum $E_{0}[24,25]$, i.e., at $E-E_{0}=0 \mathrm{eV}$ in Fig. 3.

With increasing energy $E$ above $E_{0}$, both decay rates increase linearly. However, as can be seen in Fig. 3, the minority-spin slope is twice as large as the slope for majority-spin electrons $\left[d \Gamma^{\downarrow} / d E=0.25 \pm 0.04(\mathrm{eV} \mathrm{fs})^{-1}\right.$ vs $\left.d \Gamma^{\dagger} / d E=0.12 \pm 0.02(\mathrm{eV} \mathrm{fs})^{-1}\right]$. This large difference in the energy dependence of the decay rates for electron states with opposite spin projections is the most important experimental finding of this work.

On the nonmagnetic $\mathrm{Cu}(100)$ surface the increase of decay rate with increasing energy above $E_{0}$ is due to interband scattering and intraband scattering [processes 2 and 3 in Fig. 2(a)]: The electrons gain additional phase space for decay (hatched area in Fig. 2) [23,26]. Likewise in iron, this additional phase space is comprised of the two-dimensional image-potential band (constant density of states) and the $s p$ bulk bands at energies $E>E_{0}$ [cf. Figure 2(b)]. While this will lead to an increase of the decay rate, the additional phase space is hardly spin dependent and therefore cannot account for the large difference observed in the slope $d \Gamma / d E$ between minority and majority electrons.

This difference originates from the spin asymmetry of magnon emission driven by hot electron decay. In a spinflip scattering event the excited electron occupies initial and final states corresponding to opposite spin projections. Since the spin-flip transition is associated with a change of the spin angular momentum of the hot electron, a direct 


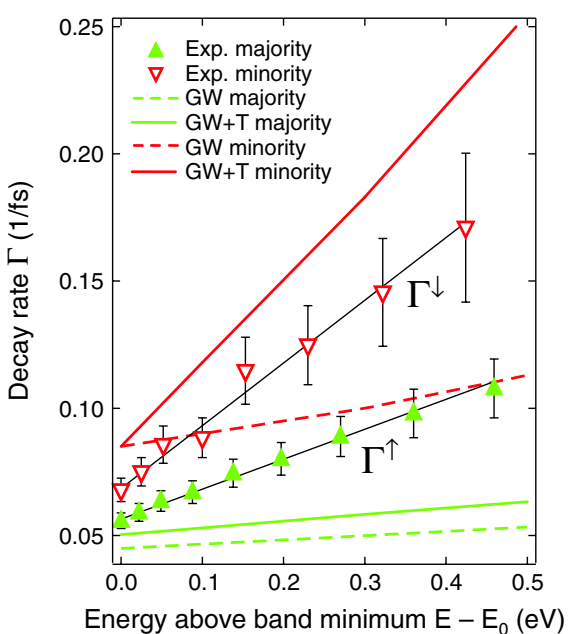

FIG. 3 (color online). Decay rate $\Gamma=1 / \tau$ of the first imagepotential states on the iron film in the majority- and minorityspin band for increasing energy above the band minimum. The measured decay rates $\Gamma^{\uparrow \downarrow}$ (symbols) are compared with the decay rates of bulk electrons calculated in the $G W$ (dashed lines) and $\mathrm{GW}+T$-matrix (solid lines) formalisms.

transition is allowed only under the influence of spin-orbit coupling. In our case spin-orbit coupling is very weak and direct spin-flip processes have negligible contribution to the decay rate. There is, however, another scenario leading to the change of the spin projection of the hot electron. This is an exchange process where the hot electron interacts with the electrons of the film. Such a process is depicted in Fig. 4(a). At the end, the excited electron has opposite spin projection with a smaller energy and, simultaneously, there is a compensating spin-flip process in the film. The latter can be either a single electron spin reversal (Stoner excitation) or a collective electron excitation (spin wave). In general, both, Stoner excitation and magnon can be emitted and absorbed in the exchange-scattering process within the constraints of energy and momentum conservation. Because of the low temperature of our experiment, we can neglect absorption. Since the Stoner excitations have characteristic energies of the order of the exchange splitting of $2.5 \mathrm{eV}$, they will not play a role in the decay processes 2 and 3 with energy transfers below $0.5 \mathrm{eV}$. In this energy range magnons can play a dominant role in the lifetime of minority electrons [14], since the magnon spectrum starts at zero energy. We demonstrate in the following that magnon emission is responsible for our observations.

In Fig. 4(c) we show the calculated spin-flip spectrum of the film. There are three spin-wave branches: one acoustic starting with zero energy and two optical with activation energies around $0.1 \mathrm{eV}$. The characteristic form of the spectrum with the parabolic dispersion of optical magnons is a consequence of the exchange interactions in the film that are stronger within than between the atomic planes. The calculations are based on a new implementation of the linear response density functional theory (LRDFT) $[27,28]$. LRDFT allows us to determine the transverse magnetic susceptibility $\chi\left(q_{\|}, \omega\right)$ of the film, taking into account our calculated band structure and thus the influence of the surface and the hybridization of the electronic states in the iron layers with the nonmagnetic substrate. Because of the nearly half-metallic character of the film the number of Stoner states in the spin-wave energy region is small and the magnon peaks are well defined [28].

As energy $\hbar \omega$ and momentum $q_{\|}$are transferred to the magnon, the conservation laws imply $\hbar \omega\left(q_{\|}\right)=E_{\downarrow}\left(k_{\|}\right)-$ $E_{\uparrow}\left(k_{\|}-q_{\|}\right)$. Considering the dispersion relations of electrons and spin waves, for a minority electron residing at the band minimum ( $\bar{\Gamma}$ point $)$ the phase space available for decay with magnon emission is severely limited. Since the spin splitting of the image-potential-state band is smaller than the activation energy of the optical spin waves, only decay into acoustic magnons is possible. Moreover, energy and momentum conservation restricts the number of available acoustic magnons to those with $q_{0} \approx 0.09 \AA^{-1}$ [cf. Fig. 4(b)]. As the momentum of the primary electron $k_{\|}$rises, there are more accessible acoustic magnon states [illustrated in Fig. 4(b) for $k_{\|} \approx 0.1 \AA^{-1}$ by the ring $q_{1}$ ]. Additionally, new decay channels open, (a)

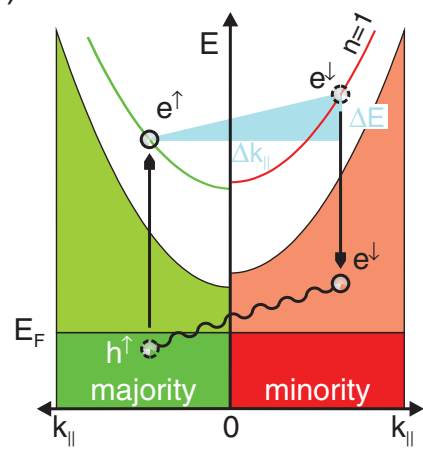

(b)

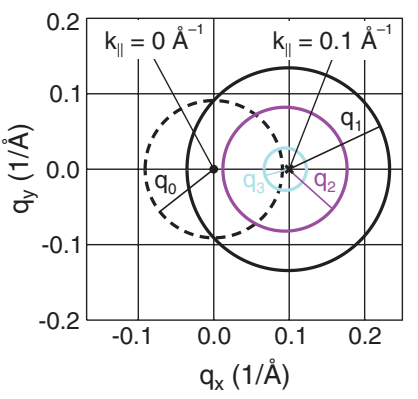

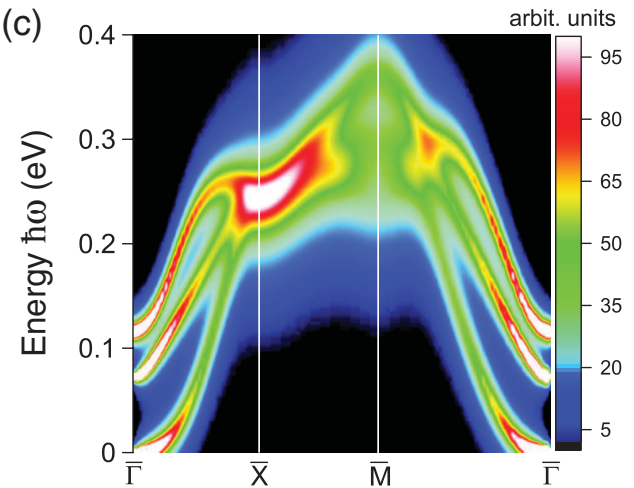

FIG. 4 (color). (a) Schematic illustration of an exchange spin-flip process. (b) Spin-wave states accessible by decaying imagepotential-state minority electrons for initial momenta $k_{\|}=0 \AA^{-1}$ (dashed line) and $k_{\|}=0.1 \AA^{-1}$ (solid lines) along $\bar{\Gamma} \bar{X}$. Rings $q_{0}$ and $q_{1}$ denote final magnon states in the acoustic mode, $q_{2}$ and $q_{3}$ in the low and high energy optical branches, respectively. (c) Spectral power $\mathfrak{s} \chi\left(q_{\|}, \hbar \omega\right)$ of spin-flip excitations in 3 monolayer iron on $\mathrm{Cu}(100)$ obtained from LRDFT calculations. 
corresponding to the generation of optical spin waves at the smaller momenta $q_{2}$ and $q_{3}$. This increase in phase space explains why, with the onset of magnon emission, the decay rate of the minority electrons increases more steeply with their initial energy.

In the framework of many-body theory, the decay rate of an excited electron can be derived from the imaginary part of the electron self-energy $\Sigma$ as $\Gamma=-2 \hbar^{-1} \operatorname{Im} \Sigma$. Different approximations for $\Sigma$ account for different excitations in the system that compensate the electron's transition. We performed calculations within two schemes: In the socalled $G W$ approximation to $\Sigma$ collective excitations of the spin-wave type are not taken into account. Only singleparticle spin-flip excitations (Stoner excitations) are incorporated. In the scheme we refer to as $G W+T$ ( $G W$ plus $T$ matrix), multiple scattering of the Stoner's electron-hole pairs is included. In this formalism the latter gives rise to spin-wave excitations. The decay rates of bulk electrons calculated via the $G W$ and $G W+T$ approaches at excitation energies corresponding to the energy losses in intraband and interband decay (processes 2 and 3) are shown as dashed and solid lines in Fig. 3, respectively. The decay rates have been scaled by the bulk penetration of the image-potential states at the band bottom and are multiplied by $2 / 3$ to account for the two-dimensionality of the thin iron film. The comparison between $G W$ and $G W+T$ confirms that magnon emission at low excitation energies yields a dominating contribution to the decay rate $\Gamma^{\downarrow}$ [13] and provides a much higher slope $d \Gamma^{\downarrow} / d E$ for spinminority electrons. Our considerations show that the physical mechanism leading to the different energy dependence of $\Gamma^{\uparrow}$ and $\Gamma^{\downarrow}$ is the emission of spin waves by the minority electrons.

The emission of magnons must take place on the femtosecond time scale, since the lifetime of these hot electrons is in the femtosecond range and the contribution of magnon emission to the decay rate of spin-down electrons is large. This establishes the time scale it requires to create a magnon in an itinerant ferromagnet. It is independent of the electronic state used to probe magnon emission, whether surface or bulk state. Our theoretical studies for bulk iron corroborate the magnon emission time at the scale of the lifetime of hot electrons.

The efficiency of magnon generation by hot electrons, on the other hand, depends on the accessibility of the spinwave states ruled by energy and momentum conservation and is therefore material dependent. This dependence has been observed for the $3 d$ ferromagnets [13,29]. Extending our combined approach to Co films, first results show an increase of $\Gamma$ with a weaker spin dependence. In contrast to the Fe films, here the exchange coupling is of comparable magnitude in and between the layers. Therefore the optical magnons in the Co films have much higher energies and cannot contribute to the decay of electrons at low excitation energies.

In conclusion, we found that the increase of the decay rate of image-potential-state electrons with increasing energy is highly spin dependent in iron films. This result can be explained by many-body theory of the excited electron lifetime only if magnon generation is taken into account. We have established that the generation of collective magnetic excitations takes place on a femtosecond time scale. Hot electrons are assumed to drive ultrafast magnetization dynamics through spin-flip electron scattering [11]. Here we show that magnon emission provides a significant microscopic source for ultrafast spin-flip processes. We could capture microscopic details of electronmagnon coupling uncovering which electrons and magnons are involved. Our results indicate that tuning the spin-selective decay rate of injected electrons could be achieved through engineering of the acoustical and optical branches of the spin-wave dispersion via the material and structural properties of the thin film.

Financial support by the Deutsche Forschungsgemeinschaft, UPV/EHU (Grant No. IT-366-07), and MCyT (Grant No. FIS2007-66711-C02-01) is gratefully acknowledged.

*anke.schmidt@uni-muenster.de

[1] R. Huber et al., Nature (London) 414, 286 (2001).

[2] M. Hase et al., Nature (London) 426, 51 (2003).

[3] R. Vollmer et al., Phys. Rev. Lett. 91, 147201 (2003).

[4] J. Schäfer et al., Phys. Rev. Lett. 92, 097205 (2004).

[5] T. Balashov et al., Phys. Rev. Lett. 97, 187201 (2006).

[6] J. Stöhr and H.-C. Siegmann, Magnetism. From Fundamentals to Nanoscale Dynamics (Springer, Berlin, 2006).

[7] E. Beaurepaire et al., Phys. Rev. Lett. 76, 4250 (1996).

[8] C. D. Stanciu et al., Phys. Rev. Lett. 99, 047601 (2007).

[9] J.-Y. Bigot, M. Vomir, and E. Beaurepaire, Nature Phys. 5, 515 (2009).

[10] B. Koopmans et al., Phys. Rev. Lett. 85, 844 (2000).

[11] B. Koopmans et al., Nature Mater. 9, 259 (2010).

[12] M. Battiato, K. Carva, and P. M. Oppeneer, Phys. Rev. Lett. 105, 027203 (2010).

[13] V.P. Zhukov, E. V. Chulkov, and P. M. Echenique, Phys. Rev. Lett. 93, 096401 (2004).

[14] J. Hong and D. L. Mills, Phys. Rev. B 62, 5589 (2000).

[15] P. M. Echenique and J. B. Pendry, J. Phys. C 11, 2065 (1978).

[16] P. M. Echenique et al., Surf. Sci. Rep. 52, 219 (2004).

[17] M. Weinelt et al., Prog. Surf. Sci. 82, 388 (2007).

[18] J. Güdde et al., Science 318, 1287 (2007).

[19] J. Thomassen et al., Phys. Rev. Lett. 69, 3831 (1992).

[20] A. B. Schmidt et al., Phys. Rev. Lett. 95, 107402 (2005).

[21] K. Blum, Density Matrix Theory and Applications (Plenum, New York, 1981).

[22] T. Hertel et al., Phys. Rev. Lett. 76, 535 (1996).

[23] M. Weinelt, J. Phys. Condens. Matter 14, R1099 (2002).

[24] F. Passek et al., Phys. Rev. Lett. 75, 2746 (1995).

[25] M. Aeschlimann et al., Phys. Rev. Lett. 79, 5158 (1997).

[26] W. Berthold et al., Phys. Rev. Lett. 88, 056805 (2002).

[27] E. K. U. Gross and W. Kohn, Phys. Rev. Lett. 55, 2850 (1985).

[28] P. Buczek et al., Phys. Rev. Lett. 102, 247206 (2009).

[29] W. X. Tang et al., Phys. Rev. Lett. 99, 087202 (2007). 